\title{
Correlation between hump dimensions and side deviation in idiopathic scoliosis before and after a conservative treatment AG Aulisa*1, V Guzzanti ${ }^{1}$, C Perisano ${ }^{2}$, M Giordano $^{1}$, ME Ricciarini ${ }^{2}$ and L Aulisa ${ }^{2}$
}

\author{
Address: ${ }^{1}$ Orthopaedics and Traumatology Department Bambino Gesù Children's Hospital, Rome, Italy and ${ }^{2}$ Rome Institute of Scientific Research \\ Orthopaedics and Traumatology Department Catholic University, P.zza s. Onofrio 4, 00165 Rome, Italy \\ Email: AG Aulisa* - agaulisa@tiscali.it \\ * Corresponding author
}

from 6th International Conference on Conservative Management of Spinal Deformities

Lyon, France. 21-23 May 2009

Published: 14 December 2009

Scoliosis 2009, 4(Suppl 2):O37 doi:10.1 186/1748-7161-4-S2-O37

This abstract is available from: http://www.scoliosisjournal.com/content/4/S2/O37

(C) 2009 Aulisa et al; licensee BioMed Central Ltd.

\section{Background}

The purposes of our study are to verify a potential correlation between hump dimensions and scoliosis curve severity and to evaluate how treatment can influence the main characteristic parameters.

\section{Methods}

134 patients (13 males and 121 females) with an average age of $12.83 \pm 1.93$ years (range 6-18 years) affected by adolescent idiopathic scoliosis (AIS) were treated with bracing until complete skeletal maturity (72 Lionese, 41 P.A.S.B., 4 Milwaukee -13 mixed treatment P.A.S.B. + lionese, 3 Milwaukee+Lionese, 1 P.A.S.B.+Milwaukee). Evaluation of treatment progress took into account two parameters: the hump (clinically measured with a humpmeter) and the Cobb angle (measured by x-rays of whole spine under load). Measurements were taken at the beginning and end of the treatment. Statistical analysis was performed using non parametrical tests to compare averages and make linear regressions between parameters. The same evaluations were made later, dividing the whole group into 4 sub-groups: patients with lumbar curves (66), thoracic curves (68), patients with age over 14 years (45), and under 13 years (89).

\section{Results}

Results demonstrated a correlation between hump and curve severity (significance was lower than $\mathrm{p}=0.001$ at the beginning and end of the treatment). Higher curve severity corresponded to a higher hump dimension. Furthermore, the effectiveness of the orthosis treatment to correct the curve severity and remodel the hump was highlighted. Treatment started with a mean Cobb angle of $29.41^{\circ} \pm$ $8.53^{\circ}$ and ended at $19.29^{\circ} \pm 9.84^{\circ}$. Hump dimensions began with a mean value of $11.61 \pm 5.59 \mathrm{~mm}$ and ended with a mean value of $6.19 \pm 4.61 \mathrm{~mm}$. It was also noticed that the degree of hump correction was greater than the correction of the curve registered in Cobb degrees. In particular, this was more noticeable in thoracic curves and in patients younger than 13 years of age.

\section{Conclusion}

The hump is the effect of rotation of a scoliosis curve. At the thoracic level, the rib hump is covered and therefore averaged by the scapula, and for this reason, there is a less important correlation between increasing rib hump and increasing spinal deformity. Orthotic treatment of idiopathic scoliosis fixes the spine deformity and is also very effective at remodeling the rib hump. This phenomenon is more noticeable when viewing the patient from behind, where the main influence has been on the rib cage. 